\title{
HRCT in primary pulmonary lymphoma: can CT imaging phenotypes differentiate histological subtypes between mucosa-associated lymphoid tissue (MALT) lymphoma and non-MALT lymphoma?
}

\author{
Yinan Chen ${ }^{1 \#}$, Aiping Chen ${ }^{2 \#}$, Hailin Jiang ${ }^{3 \#}$, Yuxuan Zhang ${ }^{4}$, Lin Zhu $^{5}$, Chunyan Xia ${ }^{6}$, Hong Yu \\ ${ }^{1}$ Department of Radiology, Shanghai Chest Hospital, Shanghai Jiaotong University, Shanghai 200030, China; ${ }^{2}$ Department of Radiology, the First \\ Affiliated Hospital of Nanjing Medical University, Nanjing 210029, China; ${ }^{3}$ Department of Interventional Radiology, Changzheng Hospital, Second \\ Military Medical University, Shanghai 200003, China; ${ }^{4}$ School of Pharmacy, Queen's University Belfast, Medical Biology Centre, Belfast, UK; \\ ${ }^{5}$ Department of Imaging and Nuclear Medicine, ${ }^{6}$ Department of Pathology, Changzheng Hospital, Second Military Medical University, Shanghai \\ 200003, China; ${ }^{7}$ Department of Radiology, Shanghai Oriental Hospital Affiliated Tongji University, Shanghai 200120, China \\ Contributions: (I) Conception and design: H Yu; (II) Administrative support: H Yu, CY Xia; (III) Provision of study materials or patients: YN Chen, \\ AP Chen, HL Jiang, CY Xia, YX Zhang, L Zhu; (IV) Collection and assembly of data: YN Chen, YX Zhang, YX Zhang, L Zhu; (V) Data analysis \\ and interpretation: YN Chen, AP Chen, HL Jiang; (VI) Manuscript writing: All authors; (VII) Final approval of manuscript: All authors. \\ \#These authors contributed equally to this work. \\ Correspondence to: Hong Yu, MD, PhD. Department of Radiology, Shanghai Oriental Hospital Affiliated Tongji University, No. 1800 Yuntai Road, \\ Pudong New Area, Shanghai 200120, China. Email: yuhongphd@163.com.
}

Background: Primary pulmonary lymphoma (PPL) mainly comprises mucosa-associated lymphoid tissue (MALT) lymphoma as well as other subtypes of lymphoma. Different phenotypes of PPL demonstrate various high-resolution computed tomography (HRCT) features. We aimed to evaluate the value of HRCT in the diagnosis and differential diagnosis of PPL, especially between MALT lymphoma and non-MALT lymphoma and the correlation between CT and pathological features.

Methods: We performed a retrospective analysis on 72 patients with PPL confirmed by pathology between 2007 and 2016. We compared the CT characteristics and correlation with pathological findings between MALT lymphoma and non-MALT lymphoma groups.

Results: All 72 patients with PPL were classified into two groups: low-grade MALT lymphoma (MALToma) (56/72) and high-grade non-MALT lymphoma (non-MALToma) (16/72). The latter group consisted of diffuse large B cell lymphoma (8/72), Hodgkin's lymphoma (3/72), T-cell lymphoma (4/72), and intravascular large B-cell lymphoma (1/72). A total of 168 lesions were analyzed, including 57 cases with multiple lesions and 15 cases with single lesion. The manifestation of four distribution patterns: nodular or mass-like involvement pattern, diffuse interstitial lung disease (DILD) pattern, pneumonia-like consolidative pattern and mixed pattern was not significantly different between MALToma and non-MALToma (all P>0.05). Signs of air bronchogram and CT angiogram occurred significantly more often in individuals with MALToma group than those with non-MALToma ( $75 \%$ vs. $25 \%, \mathrm{P}=0.001 ; 64.3 \%$ vs. $12.5 \%, \mathrm{P}<0.001$; respectively). Conversely, the halo sign presented more often in non-MALToma than in MALToma patients $(19 \%$ vs. $63.6 \%, \mathrm{P}=0.02)$. In addition, the butterfly sign was only observed in four patients with MALToma.

Conclusions: HRCT imaging phenotypes were beneficial in the diagnosis of PPL. Solitary or multifocal nodules/ masses and consolidation were the most common imaging patterns. The air bronchogram sign, CT angiogram sign, halo sign, and butterfly sign could be potential to help to differentiate MALToma from non-MALToma.

Keywords: Pulmonary lymphoma; mucosa-associated lymphoid tissue lymphoma (MALT lymphoma); computed tomography (CT); diagnosis; pathology

Submitted Mar 12, 2018. Accepted for publication Sep 18, 2018.

doi: $10.21037 /$ jtd.2018.10.63

View this article at: http://dx.doi.org/10.21037/jtd.2018.10.63

(C) Journal of Thoracic Disease. All rights reserved.

jtd.amegroups.com

7 Thorac Dis 2018;10(11):6040-6049 


\section{Introduction}

Primary pulmonary lymphoma (PPL) is defined as clonal lymphoid proliferation involving lung parenchyma and/ or bronchi without detectable extra pulmonary lymphoma at primary diagnosis or the subsequent 3 months (1). PPL is a rare neoplasm, accounting for $\leq 1 \%$ of all lymphomas and $0.5-1 \%$ of primary pulmonary malignancies (2). The most common type of PPL is mucosa-associated lymphoid tissue (MALT) lymphoma (70-90\%) (3), which is also called extranodal marginal zone B cell lymphoma. It can arise from any mucosal site. However, lung is the most common organ involved especially MALT of the bronchus. The second most frequent histological type of PPL is diffuse large B-cell lymphoma (DLBCL, 5-20\%) (4). In 1963, Saltzstein (5) was the first to report a large number of PPLs, since then, a variety of studies have reported the different subtypes of PPL. While the majority focused on MALToma, few have reported on other histological phenotypes of PPL.

The radiographic findings of PPL are nonspecific including solitary nodule, multiple ill-defined nodules, consolidated mass with air bronchogram, atelectasis, bronchiectasis and bronchiolitis and ground glass opacity $(6,7)$. Therefore, PPL can be easily misdiagnosed as other lung diseases such as lung cancer, pulmonary metastases, multiple pneumonia, variant of organizing pneumonia, etc. (2). Several studies had been conducted to develop characteristic computed tomography (CT) features for diagnosis and differential diagnosis of PPL, but to our best knowledge, CT characteristics of MALT lymphoma versus non-MALT lymphoma have not been evaluated (2,8-10).

Given the different degrees of pathological differentiation and therapy, it is crucial to identify pulmonary MALT lymphoma with low-grade features and other PPLs with high-grade histological subtypes (2). Usually, pulmonary MALT lymphomas are indolent and progress more slowly than other subtypes of PPL; patients with MALT lymphomas have better prognosis than those with other subtypes of malignant lymphomas. For local low-grade pulmonary MALT lymphoma, the mainstay of treatment is surgery; however, for high-grade pulmonary lymphoma, chemotherapy is the first choice of treatment. Wei et al. (11) used the rituximab plus cladribine as a first-line therapy for unresectable bronchial-associated lymphoid tissue lymphoma and determined its effectiveness and safety. Based on this fact, the identification of PPL subtypes could guide clinicians to make optimal therapy management.

In the present study, we aimed to evaluate the value of high-resolution computed tomography (HRCT) in the diagnosis and differential diagnosis of PPL, especially in differentiating MALT lymphoma and non-MALT lymphoma and analyze the correlation between CT and pathological features.

\section{Methods}

\section{Patients}

We retrospectively analyzed the data of 72 patients with pathologically confirmed PPL admitted in our hospitals between January 2007 and December 2016. Approval for the study was granted by Shanghai Changzheng Hospital's Research Ethics Committee (institutional review board number: KSY1668). The diagnosis of PPL was based on the diagnostic criteria proposed by Cordier et al. (12), including the involvement of lung/lobar/primary bronchus, with or without mediastinal involvement, and no evidence of extrathoracic lymphoma at primary diagnosis or the subsequent 3 months. The pathology results of all patients were acquired as follows: thoracoscopic wedge resection (15 cases), thoracoscopic lobectomy (19 cases), CT guided biopsy (32 cases), and thoracotomy (6 cases). The demographic and clinical characteristics of all patients were obtained from the medical records. This retrospective study was approved by our institutional review board, which waived informed consent.

\section{CT scanning}

All chest CT scans of 16, 23, and 33 patients were performed from the top of the thoracic cage to the level of bilateral adrenal glands; using the Aquilion 16 (Toshiba, Tokyo, Japan), Light Speed 64VCT (GE Healthcare, Freiburg, Germany); and Brilliance 256 (Philips Medical Systems, The Netherlands) CT scanners, respectively. Twenty-seven patients underwent a contrast-enhanced CT scan (Iopamiro 370, $90 \mathrm{~mL}$ ). Arterial phase and delayed phase images were acquired at 20-25 and 75-90 s after injection, respectively. Helical scanning protocols were as follows: $120 \mathrm{kVp}$; 50-150 mAs; beam pitch, 0.516-0.98; slice thickness, $5 \mathrm{~mm}$; matrix, $512 \times 512$. Imaging data was reconstructed into 0.625 or $1 \mathrm{~mm}$ slice thickness with a soft-tissue algorithm for mediastinal window image, and a lung algorithm for lung window image. All images were viewed with both lung window (window width, 1,500 $\mathrm{HU}$; window level, $-500 \mathrm{HU}$ ) and mediastinum window (window 
Table 1 Definition of assessed CT features

\begin{tabular}{|c|c|c|}
\hline Lesions & Feature & Definition \\
\hline \multirow{10}{*}{ Lung lesion } & Location & Right versus left; superior versus middle versus inferior lobe \\
\hline & Nodule & Round opacity, at least moderately well marginated and $\leq 30 \mathrm{~mm}$ in diameter \\
\hline & Mass & Round opacity, at least moderately well marginated and $>30 \mathrm{~mm}$ in diameter \\
\hline & * Subpleural & Consolidative lesion polygonal in shape, based pleural or interlobar fissure \\
\hline & $\begin{array}{l}\text { Lobular or } \\
\text { segmental }\end{array}$ & Consolidative lesion involved whole lobe or segment \\
\hline & * Peribronchial & $\begin{array}{l}\text { Consolidative lesion distributed along the bronchovascular bundles with a tapered } \\
\text { shape and tip towards to hilar }\end{array}$ \\
\hline & Air bronchogram sign & The air-filled trachea through the consolidation or nodular/mass lesions normally \\
\hline & CT angiogram sign & $\begin{array}{l}\text { The imaging of branch of pulmonary vascular through normally in the consolidation } \\
\text { or masses after enhancement }\end{array}$ \\
\hline & Halo sign & A solid nodule/mass surrounded by a halo of ground-glass attenuation \\
\hline & Cross lobe sign & $\begin{array}{l}\text { A consolidative lesion invading interlobular fissure distributed along multiple lung } \\
\text { lobes }\end{array}$ \\
\hline \multirow[t]{2}{*}{ Lymph node lesion } & Enlargement & Shorter diameter $>10 \mathrm{~mm}$ \\
\hline & Location & Mediastinum or hilar \\
\hline
\end{tabular}

width, $375 \mathrm{HU}$; window level, $50 \mathrm{HU})$.

\section{CT image interpretation}

All the post-processed images were interpreted by two thoracic radiologists with 15 and 10 years' experience in chest CT. The observers were blinded to the clinical and pathologic diagnosis. Decisions on CT scan findings were reached by consensus. Axial images were analyzed at lung window and mediastinum window. The images of all patients were reconstructed by multiple plane reconstruction (MPR) and volume rendering (VR) to fully show the imaging characteristics.

A series of signs defined by radiologists (Table 1) demonstrated the characteristics of lung, lymph nodes and pleura.

\section{Pathologic assessment}

Pathologists diagnosed tumors mainly by morphological and immunohistochemistry. For patients with difficult diagnosis, gene rearrangement detection was carried out. In this study, only 3 patients underwent polymerase chain reaction (PCR), 2 MALToma patients showed $\mathrm{t}(11 ; 18)$ (q21;Q21). The antibodies used in immunohistochemical staining and results are shown in Tables 2 and 3.

\section{Statistical analysis}

Continuous data were compared between two groups using Student's $t$ test or Mann-Whitney's U test, while categorical data were compared using Chi-square tests. All statistical analyses were performed in SPSS20.0 (SPSS Inc., 
Chicago, IL, USA). A P value less than 0.05 was considered statistically significant for all tests.

\section{Results}

\section{General characteristics}

The clinical characteristics of the patients are described in Table 4. Of the 72 patients, there was no gender predominance (39 women and 33 men; median age, 57 years; range, 21-76 years). Twenty-six patients who underwent routine radiographic detection of the pulmonary lesions showed no clinical symptoms. However, 26 other patients had respiratory symptoms. Twenty-nine patients who had a smoking history experienced respiratory symptoms such as cough, chest pain, and chest distress.

Table 2 Antibodies used in the immunohistochemical staining.

\begin{tabular}{ll}
\hline Antibodies & Specificity \\
\hline L26 & CD20, B-cells \\
MB2 & $\begin{array}{l}\text { Cytoplasmic antigen in B-cells except plasma } \\
\text { cells }\end{array}$ \\
MT1 & $\begin{array}{l}\text { Cortical and medullary thymocytes, lymphocytes } \\
\text { in T-cell region }\end{array}$ \\
UCHL1 & CD45RO, human T-cells \\
BerH2 & Human Ki-1 antigen, CD30 \\
ION1 & CD15, Reed-Sternberg cells \\
Bcl6 & $\begin{array}{l}\text { differential diagnosis of follicular lymphoma and } \\
\text { MALT lymphoma }\end{array}$ \\
CyclinD1 & $\begin{array}{l}\text { differential diagnosis of mantle lymphoma and } \\
\text { MALT lymphoma }\end{array}$ \\
\hline
\end{tabular}

MALT, mucosa-associated lymphoid tissue.
Seventeen individuals had only B symptoms, including fever, fatigue, and weight loss. In addition, three patients were identified to have Sjogren's syndrome. There was no significant difference in the clinical manifestations between patients with MALToma and those with non-MALToma (Table 4).

\section{Lung lesion features}

A total of 168 lesions were observed in 72 PLL patients including 57 cases with multiple lesions and 15 cases with a single lesion. The lesions distributed randomly, and there was no statistical difference between distribution in most lobes other than the right lower lobe $(\mathrm{P}=0.007$; Table 5). Based on the pattern and distribution of parenchymal abnormalities, PPL patients were classified into four groups: (I) nodular or mass-like involvement pattern (32 cases: 21 MALToma and 11 non-MALToma). The lesions presented as solitary or multiple nodules/masses (Figure 1) with clear boundary and smooth margin and no signs of lobulation or spiculation. Straight edge was observed in 5 cases, and 17 cases involved the subpleural area, but without pleural indentation; (II) pneumonia-like consolidative pattern (18 cases: 16 MALToma and 2 non-MALToma). The lesions presented consolidation in polygon-based pleura consolidation involving lobe/segment, and consolidation with tapered distribution along bronchovascular bundles (Figure 2). Fifteen cases showed clear boundary, 7 of which showed bulging fissure sign; (III) diffuse interstitial lung disease (DILD) pattern ( 3 cases: 2 MALToma and 1 nonMALToma). The lesions presented diffuse ground glass opacity with interlobular septal thickening (Figure 3). (IV) Mixed pattern (19 cases: 17 MALToma and 2 nonMALToma). These lesions include at least two patterns

Table 3 Histopathological classification and immunohistochemical findings in 72 cases of primary pulmonary lymphoma

\begin{tabular}{|c|c|c|c|c|c|c|c|c|c|c|}
\hline Classification & No. of cases & Light chain $(\kappa \lambda)$ & \multicolumn{8}{|c|}{ Immunochemistry } \\
\hline MALToma & 56 & $17-29$ & $54 / 56$ & $55 / 56$ & $3 / 37$ & $2 / 39$ & n.d & n.d & $31 * / 49$ & $28^{*} / 46$ \\
\hline DLBCL & 8 & - & $7 / 8$ & $5 / 6$ & $1 / 8$ & $0 / 8$ & $0 / 1$ & $0 / 2$ & $5 / 8$ & n.d \\
\hline $\mathrm{MCHL}$ & 3 & - & $0 / 3$ & $0 / 3$ & $1 / 3$ & $1 / 3$ & $3 / 3$ & $3 / 3$ & n.d & n.d \\
\hline IVBCL & 1 & - & $1 / 1$ & $1 / 1$ & $0 / 1$ & $0 / 1$ & $1 / 1$ & $0 / 1$ & n.d & n.d \\
\hline
\end{tabular}

*, negative expression. MALToma, mucosa-associated lymphoid tissue lymphoma; DLBCL, diffuse large B-cell lymphoma; MCHL, mixed cell Hodgkin's lymphoma; IVLBCL, intravascular large B-cell lymphoma; n.d, not done. 
Table 4 Clinicopathological features of 72 PPL patients

\begin{tabular}{|c|c|c|c|}
\hline Characteristics & Overall & MALToma & Non-MALToma \\
\hline \multicolumn{4}{|l|}{ Gender } \\
\hline Male & 33 & 28 & 5 \\
\hline Female & 39 & 28 & 11 \\
\hline Asymptomatic & 29 & 22 & 7 \\
\hline \multicolumn{4}{|l|}{ B symptoms } \\
\hline Fever & 5 & 5 & 0 \\
\hline Fatigue & 8 & 5 & 3 \\
\hline Weight loss & 4 & 3 & 1 \\
\hline \multicolumn{4}{|l|}{ Respiratory symptom } \\
\hline Cough & 15 & 11 & 4 \\
\hline Chest pain & 6 & 4 & 2 \\
\hline Chest distress & 5 & 5 & 0 \\
\hline Smoking history & 29 & 26 & 3 \\
\hline Sjogren's syndrome & 3 & 3 & 0 \\
\hline PPL & 72 & 56 & 16 \\
\hline MALT & 56 & 56 & 0 \\
\hline DLBCL & 8 & 0 & 8 \\
\hline HL & 3 & 0 & 3 \\
\hline T-cell L & 4 & 0 & 4 \\
\hline Intravascular & 1 & 0 & 1 \\
\hline
\end{tabular}

PPL, primary pulmonary lymphoma; MALT, mucosa-associated lymphoid tissue; MALToma, mucosa-associated lymphoid tissue lymphoma; DLBCL, diffuse large B-cell lymphoma; HL, Hodgkin's lymphoma.

above. The differences between two subtypes were not statistically significant (all $\mathrm{P}>0.05$; Table 5).

Some statistically different CT features included air bronchogram sign, CT angiogram sign and halo sign. In addition, the butterfly sign could only be seen in four patients with MALToma. No significant difference in cross lobe sign was present between two groups.

\section{Pleural features}

Pleural invasion, determined by pleural indentation and pleural effusion, was absent. Two cases (each with MALToma and non-MALToma) showed pleural thickening.

\section{Lymph node features}

Findings of lymphadenopathy were very rare: only one enlarged lymph node with diameter more than $1 \mathrm{~cm}$ in the MALToma group without pathology results.

\section{Pathology characteristics}

The antibodies used in the immunohistochemical staining for the analysis of PPLs are described in Table 2. Histopathological classification and immunohistochemical findings in 72 cases of PPLs are presented in Table 3. Three patients with MALToma underwent clonality in lymphoid cells by PCR assay of immunoglobulin heavy chain ( $\mathrm{IgH})$ gene rearrangement in bronchoalveolar lavage (BAL) fluid cells showed not high.

\section{Discussion}

In the present study, we assessed the distribution and features of lung lesions on HRCT and analyzed some specific signs in PPL patients. We compared two groups based on pathologically differentiated degree of PPL. The most frequent patterns of MALToma and non-MALToma were nodular/mass-like and consolidation patterns, which was consistent with previous reports on MALT and large B cell lymphomas $(9,10,13)$. These patterns were followed by the pneumonia-like consolidative and mixed pattern. DILD pattern was rare, similar to findings in a previous study (7). We also summarized some characteristic signs of PPL such as air bronchogram sign, CT angiogram sign, halo sign, cross lobe sign, butterfly sign, and across lobe sign. Typical CT signs could predict pathological features (13). Although a few studies published the CT findings of MALT and nonMALToma; and showed no significant difference between them, we surmise that the imaging features of PPL are suggestive of the histological subtypes in many cases and have a high relevance with pathologic characteristics. Our results showed that air bronchogram and CT angiogram signs were more common in patients with MALToma than those with non-MALToma. The halo sign was more frequent in patients with non-MALToma.

The air bronchogram sign is present in different types of PPL, especially those that display nodular/mass-like and pneumonia-like consolidative patterns. Photomicrograph revealed that the tumor cells infiltrated and grew along the interstitial lung and bronchial submucosa, but there were no tumor cells or destruction of the bronchial 
Table 5 Chest CT findings, patterns and features in patients with PPL

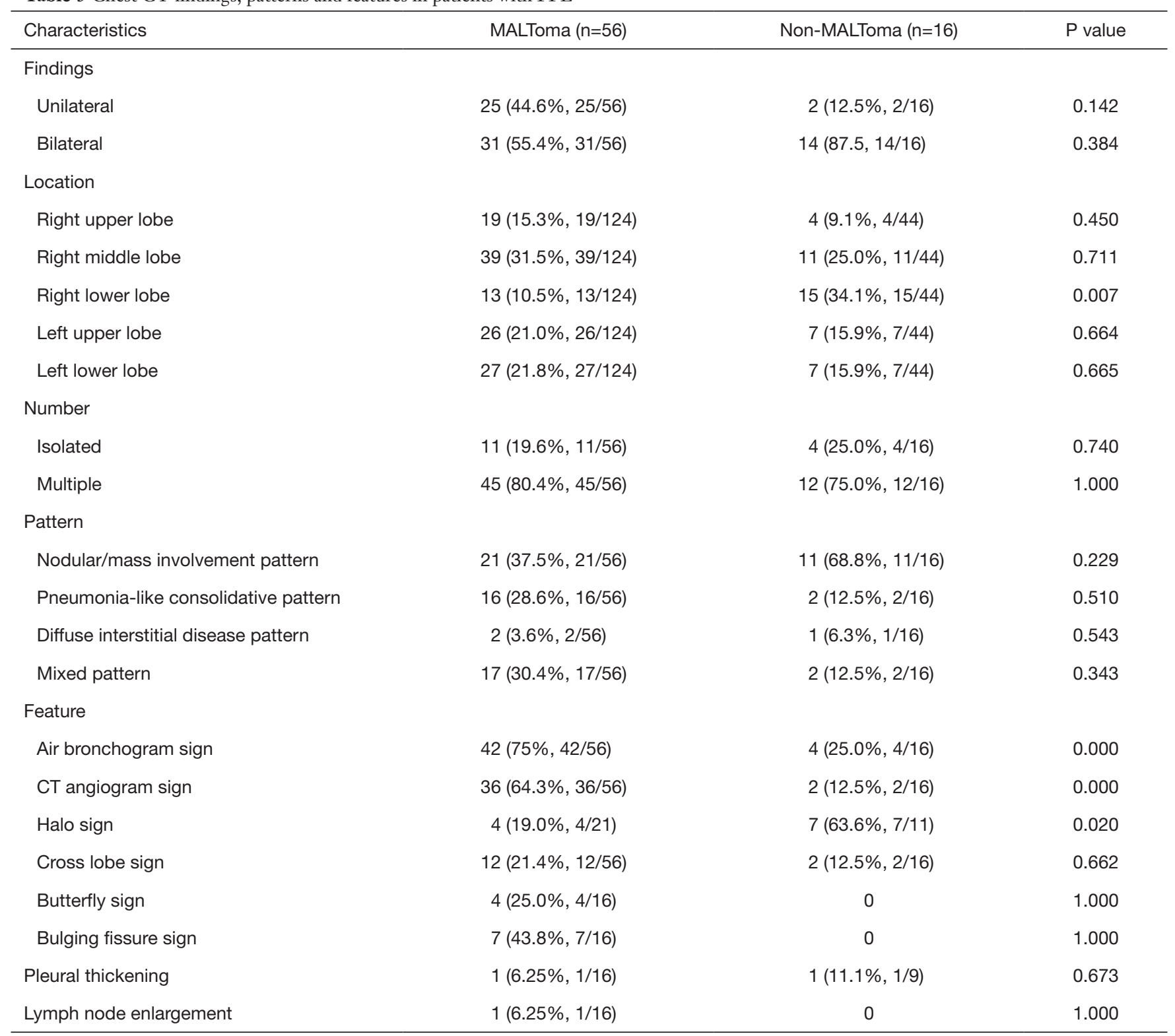

PPL, primary pulmonary lymphoma; MALToma, mucosa-associated lymphoid tissue lymphoma.

wall (Figure 1). The air bronchogram sign is considered remarkable for pulmonary lymphoma and is more frequent in patients with MALToma than those with non-MALToma ( $75 \%, 42 / 56$ vs. $25 \%, 4 / 16, \mathrm{P}=0.000)$. We speculate that the difference might be caused by different degrees of pathological differentiation. Primary pulmonary MALToma is a monoclonal B lymphocyte proliferation that originated from BALT. It is made up of submucosal collections of lymphocytes (B and T cells) present just beneath areas of specialized bronchial epithelium throughout the airways, and, most especially at areas of bronchial bifurcation $(14,15)$. Therefore, the tumor infiltrates along the bronchial submucosal epithelium and slowly progresses, while bronchial wall is inviolable and bronchial lumen keeps smooth. By contrast, patients with non-MALT are highly malignant lymphomas and invade the bronchial wall early and rapidly.

The CT angiogram sign is the presence of normal pulmonary vessels with pulmonary consolidation or masses after enhancement (Figure 1D). It is initially reported as 

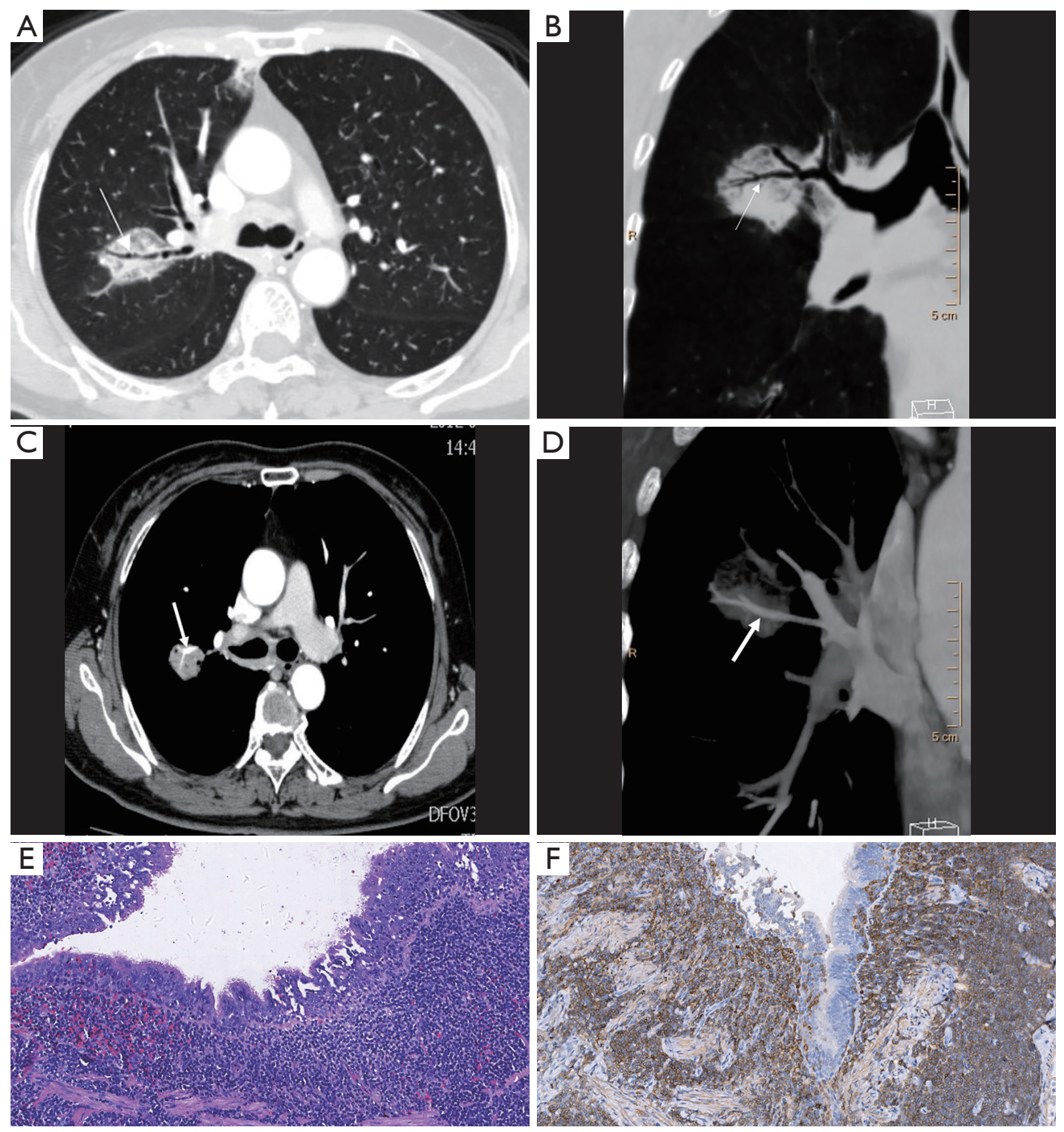

Figure 1 Marginal zone B-cell lymphoma of bronchus associated lymphoid tissue (BALT) in a 62-year-old woman. (A,B) Axial lung-window CT scan and multiplanar reconstruction (MPR) show lung nodule with air bronchogram (white arrows) in the posterior segment of right upper lobe. (C,D) Axial mediastinum-window CT scan and maximum intensity projection (MIP) show CT angiogram sign (white arrows). (E) High-magnification photomicrograph reveals a dense cuff of lymphoid cells along bronchial submucosa (hematoxylin-eosin, $\times 200$ ). (F) Immunohistochemical staining confirmed MALT lymphoma (positive CD79 $\alpha, \times 400$ ).

a specific sign for bronchioloalveolar cell carcinoma and demonstrates an intact vascular network within consolidated lung of low attenuation (16). A previous study has identified this sign in PPL patients (10). The sign occurred more frequently in patients with MALToma than those with non-MALToma $(64.3 \%, 36 / 56$ vs. $12.5 \%, 2 / 16, \mathrm{P}=0.000)$. We speculate that the difference is due to differences in their biological malignancy. MALToma infiltrates along the peribronchovascular interstitium without invading the vascular wall. However, the high-grade malignant lymphoma develops rapidly and can invade the pulmonary vessels at an early stage (15). We speculate that this sign might contribute to the diagnosis and differential diagnosis of PPL and lung cancer in different stage, because advanced stage lung cancer could infiltrate pulmonary vessels and vascular wall to cause vascular distortion and destruction.

The halo sign, indicates ground glass attenuation surrounding a pulmonary nodule on CT imaging. It is of clinical significance in the early diagnosis of invasive pulmonary aspergillosis (IPA), which is characterized 

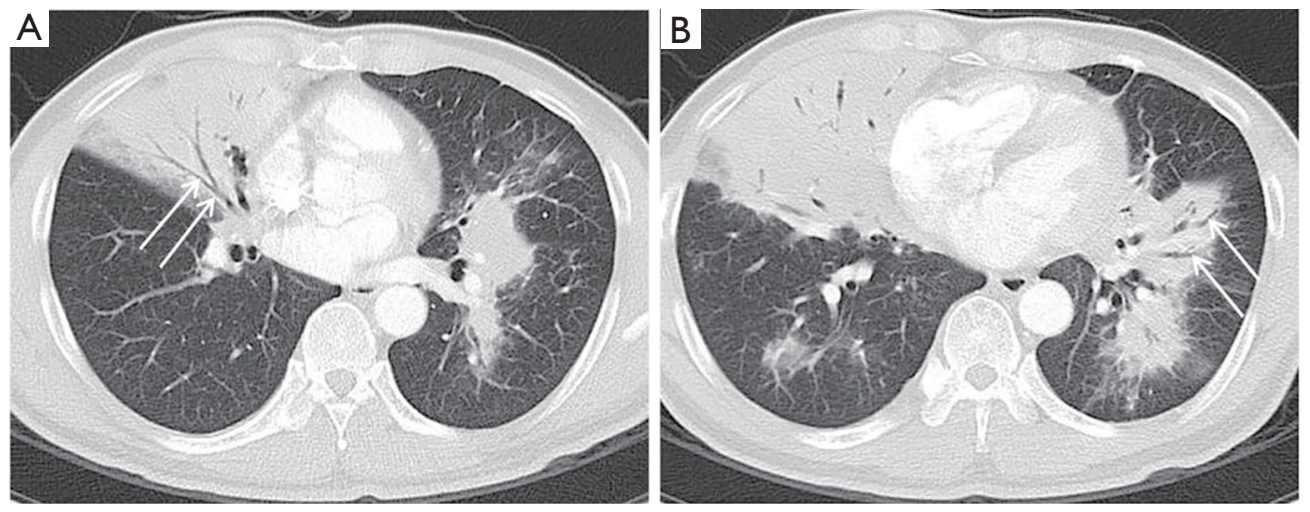

Figure 2 BALT in a 48-year-old man. (A,B) Transverse lung window CT scans (5-mm section thickness) shows pneumonia-like consolidative pattern with bilateral multiple lobar and patchy consolidations containing air bronchogram sign (white arrows). Perihilar consolidation along bronchovascular bundles in both left upper and lower lobes across oblique fissure, similar to butterfly. BALT, bronchus associated lymphoid tissue.
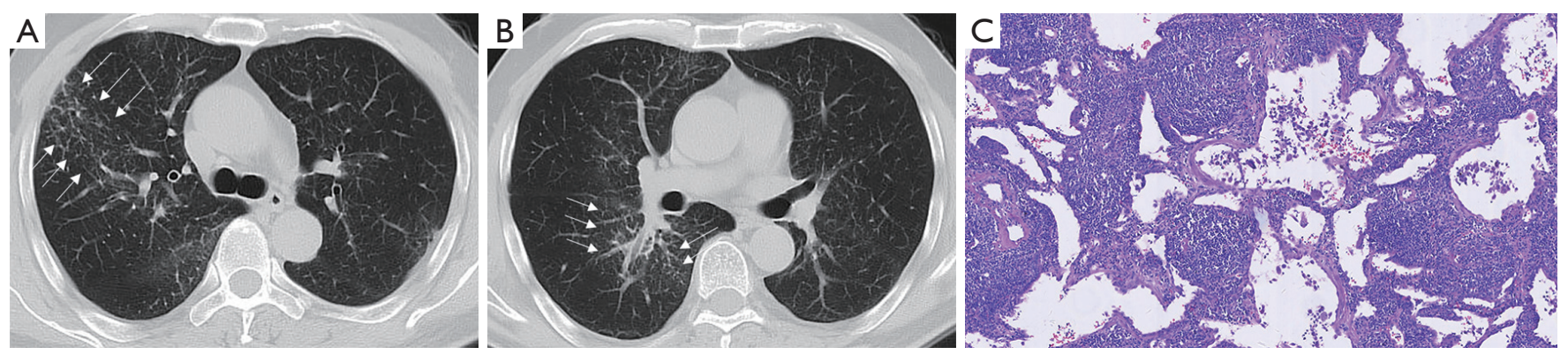

Figure 3 BALT in a 62-year-old man. (A,B) Transverse lung-window CT scan (5-mm section thickness) shows diffuse ground glass opacity with interlobular septal thickening in bilateral parenchyma (white arrows). (C) Diffuse infiltration of tumor cells under interlobular septum (hematoxylin-eosin, $\times 100$ ). BALT, bronchus associated lymphoid tissue.

pathologically by foci of infarction surrounded by alveolar hemorrhage (17). In pulmonary lymphoma, microscopy manifestation represents an infiltration of the surrounding interstitium by less densely arranged tumor cells, relating to the invasiveness of PPL. In a study conducted by Kawel et al. (18), the halo sign occurred more significantly in IPA than in PPL, which might be explained by their different invasive mechanisms and incidence as well as different onset time and degree of disease progression. In our study, this sign occurred more frequently in patients with nonMALToma than in those with MALToma (63.6\%, 7/11 vs. $19 \%, 4 / 21, \mathrm{P}=0.020)$. Although non-specific, the sign may be related to the higher degree of interstitium invasiveness of non-MALToma than of MALToma.

A total of 16 cases in our study presented that lung lesions crossed lobe fissures and infiltrated to the adjacent lobe on CT, and the distribution from the hilum to the periphery across lobe fissures is defined as butterfly sign. We speculate that the mechanism of this sign is due to specific lymphangitic spread along pleura, interlobular septa, and bronchovascular bundles (13). In the MALToma group, four patients presented the butterfly sign accompanied with across lobe distribution (Figure 2). This sign might give a clue to differential diagnosis of other pulmonary malignant tumors especially lung cancer. The bulging fissure sign was detected in seven patients with pneumonia-like consolidative pattern $(38.9 \%, 7 / 18)$, it refers to lobar consolidation where the affected portion of the lung is expanded to cause displacement of the adjacent fissure. Usually, the presence of a bulging fissure 
sign is suggestive of Klebsiella pneumoniae infection (19). Moreover, central lung cancer, especially with atelectasis, is also easy to show this sign. Yamakawa et al. (20) reported a rare case of pulmonary Hodgkin's lymphoma presenting this sign. Therefore, it is nonspecific for benign and malignant diseases, recognization of this sign can remind clinicians to think of the differential diagnosis of PPL in clinical work.

A defining feature of PPL in this and other studies was the absence of radiographically significant hilar or mediastinal adenopathy. In our study, only one case presented mediastinal and hilar lymph nodes enlargement. This is non-consistent with part of the published results $(21,22)$. However, in a study that included patients with high stages of disease, mediastinal lymphadenopathy was not frequently observed (23). In most researchers' view, according to the diagnostic criteria, PPLs are essentially extranodal lymphomas, hilar and mediastinal lymphadenopathy is rare in the early stage. These different results may be caused by the data bias and population and geographical differences. Moreover, pleural invasion, as indicated by pleural indentation and pleural effusion, was absent (23).

There are some limitations in our study. First of all, this is a retrospective study. Some patients only underwent plain CT scan, and contrast-enhanced CT information was lacking. Therefore, we can hardly analyze patients' blood supply characteristics. Second, the biomarkers of histopathologic exams were incomplete, so we could not compare the imaging differences of phenotypes with plasmacytic differentiation and those without. In addition, although the number of PPL patients in the present study was larger than that of any previous report, the small sample size of non-MALT lymphoma patients might limit the accuracy and reliability of the results. Therefore, further investigations with a larger cohort of PPL patients are necessary to confirm out results.

\section{Conclusions}

PPL manifests diverse distribution patterns of lung abnormality on CT scan. Solitary or multifocal nodules/ masses and consolidation are the most common imaging patterns. Besides the persistent and indolent nature of lesions, some significant CT signs, including air bronchogram sign, CT angiogram sign, halo sign, cross lobe sign, and butterfly sign, could be considered as suggestive for identification of PPL, and to differentiate histological subtypes between MALT and non-MALT lymphoma.

\section{Acknowledgements}

Funding: This study was supported by National Natural Science Foundation of China (code: 81671697); Shanghai Municipal Science and Technology Development Fund (code: 15411952000); and Shanghai Hospital Development Center Foundation (code: SHDC12014227), and Shanghai Hospital Development Center Foundation (code: SHDC22015033).

\section{Footnote}

Conflicts of Interest: The authors have no conflicts of interest to declare.

Ethical Statement: This retrospective study was approved by Shanghai Changzheng Hospital's Research Ethics Committee (institutional review board number: KSY1668).

\section{References}

1. Hu YH, Hsiao LT, Yang CF, et al. Prognostic factors of Chinese patients with primary pulmonary non-Hodgkin's lymphoma: the single-institute experience in Taiwan. Ann Hematol 2009;88:839-46.

2. Cardenas-Garcia J, Talwar A, Shah R, et al. Update in primary pulmonary lymphomas. Current opinion in pulmonary medicine 2015;21:333-7.

3. Cadranel J, Wislez M, Antoine M. Primary pulmonary lymphoma. Eur Respir J 2002;20:750-62.

4. Wei S, Li X, Qiu X, et al. Primary lung lymphoma involving the superior vena cava. World J Surg Oncol 2012;10:131.

5. Saltzstein SL. Pulmonary malignant lymphomas and pseudolymphomas: classification, therapy, and prognosis. Cancer 1963;16:928-55.

6. Lee DK, Im JG, Lee KS, et al. B-cell lymphoma of bronchus-associated lymphoid tissue (BALT): CT features in 10 patients. J Comput Assist Tomogr 2000;24:30-4.

7. Bae YA, Lee KS, Han J, et al. Marginal zone B-cell lymphoma of bronchus-associated lymphoid tissue: imaging findings in 21 patients. Chest 2008;133:433-40.

8. Graham BB, Mathisen DJ, Mark EJ, et al. Primary pulmonary lymphoma. Ann Thorac Surg 2005;80:1248-53.

9. Albano D, Borghesi A, Bosio G, et al. Pulmonary mucosa-associated lymphoid tissue lymphoma: 18F-FDG PET/CT and CT findings in 28 patients. Br J Radiol 2017;90:20170311. 
10. King LJ, Padley SP, Wotherspoon AC, et al. Pulmonary MALT lymphoma: imaging findings in 24 cases. Eur Radiol 2000;10:1932-8.

11. Wei Z, Li J, Cheng Z, et al. A single center experience: rituximab plus cladribine is an effective and safe first-line therapy for unresectable bronchial-associated lymphoid tissue lymphoma. J Thorac Dis 2017;9:1081.

12. Cordier JF, Chailleux E, Lauque D, et al. Primary pulmonary lymphomas. A clinical study of 70 cases in nonimmunocompromised patients. Chest 1993;103:201-8.

13. Sirajuddin A, Raparia K, Lewis VA, et al. Primary pulmonary lymphoid lesions: radiologic and pathologic findings. Radiographics 2016;36:53-70.

14. Bienenstock J, McDermott MR. Bronchus- and nasalassociated lymphoid tissues. Immunol Rev 2005;206:22-31.

15. Fiche M, Caprons F, Berger F, et al. Primary pulmonary nonHodgkin's lymphomas. Histopathology 1995;26:529-37.

16. Im JG, Han MC, Yu EJ, et al. Lobar bronchioloalveolar carcinoma:" angiogram sign" on CT scans. Radiology 1990;176:749-53.

17. Lee YR, Choi YW, Lee KJ, et al. CT halo sign: the

Cite this article as: Chen Y, Chen A, Jiang H, Zhang Y, Zhu L, Xia C, Yu H. HRCT in primary pulmonary lymphoma: can CT imaging phenotypes differentiate histological subtypes between mucosa-associated lymphoid tissue (MALT) lymphoma and non-MALT lymphoma? J Thorac Dis 2018;10(11):6040-6049. doi: $10.21037 /$ jtd.2018.10.63 spectrum of pulmonary diseases. Br J Radiol 2005;78:862-5.

18. Kawel N, Schorer GM, Desbiolles L, et al. Discrimination between invasive pulmonary aspergillosis and pulmonary lymphoma using CT. Eur J Radiol 2011;77:417-25.

19. Okada F, Ando Y, Honda K, et al. Clinical and pulmonary thin-section CT findings in acute Klebsiella pneumoniae pneumonia. Eur Radiol 2009;19:809-15.

20. Yamakawa H, Yoshida M, Katagi H, et al. Pulmonary Hodgkin's lymphoma presenting with a bulging fissure sign. Intern Med 2014;53:2021-2.

21. Pina-Oviedo S, Weissferdt A, Kalhor N, et al. Primary Pulmonary Lymphomas. Adv Anat Pathol 2015;22:355-75.

22. Wislez M, Cadranel J, Antoine M, et al. Lymphoma of pulmonary mucosa-associated lymphoid tissue: CT scan findings and pathological correlations. Eur Respir J 1999;14:423-9.

23. Kurtin PJ, Myers JL, Adlakha H, et al. Pathologic and clinical features of primary pulmonary extranodal marginal zone B-cell lymphoma of MALT type. Am J Surg Pathol 2001;25:997-1008. 